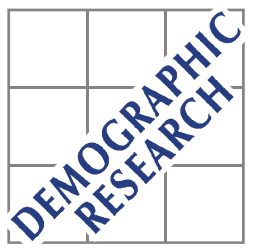

Demographic Research a free, expedited, online journal of peer-reviewed research and commentary in the population sciences published by the Max Planck Institute for Demographic Research Konrad-Zuse Str. 1, D-18057 Rostock · GERMANY www.demographic-research.org

DEMOGRAPHIC RESEARCH

VOLUME 13, ARTICLE 24, PAGES 597-614

PUBLISHED 15 DECEMBER 2005

http://www.demographic-research.org/Volumes/Vol13/24/

DOI: $10.4054 /$ DemRes.2005.13.24

Research Article

Lifesaving, lifetimes and lifetables

\author{
James W. Vaupel \\ (C) 2005 Max-Planck-Gesellschaft.
}




\section{Table of Contents}

1 Introduction $\quad 598$

2 How saving a life alters life expectancy 600

3 Individual lifetimes $\quad 602$

$4 \quad$ The triangle of turbulence $\quad 604$

$5 \quad$ How large is the distortion? 606

6 Considerations about true life expectancy 607

$7 \quad$ A model of stretched lifetimes $\quad 609$

8 Quantum and tempo vs. proportions and increments 609

9 Directions for research $\quad 610$

10 Acknowledgements 613

$\begin{array}{ll}\text { References } & 614\end{array}$ 


\title{
Lifesaving, lifetimes and lifetables
}

\author{
James W. Vaupel ${ }^{1}$
}

\begin{abstract}
Mortality change roils period rates. In the short term, conventional calculations of agespecific probabilities of death and life expectancy in the period immediately after the change depend on how many lives have been saved. In the long term, the probabilities and period life expectancy also depend on how long these lives have been saved. When mortality is changing, calculations of period life expectancy do not, except in special circumstances, measure the life expectancy of a cohort of newborns that hypothetically live all their lives under the new mortality regime.
\end{abstract}

${ }^{1}$ Max Planck Institute for Demographic Research 


\section{Introduction}

When a life is saved, how long is death averted? When death rates are declining, how should period lifetables be estimated? This demographic essay explains why and how the answer to the second question hinges on the answer to the first. My thinking was stimulated by the pathbreaking research by Bongaarts and Feeney $(2002 ; 2003 ; 2005)$ on why conventional calculations may lead to distorted estimates of period life expectancy.

The conventional formula for period life expectancy at current death rates can be expressed as

$$
e_{o}=\int_{0}^{\omega} \exp \left[-\int_{0}^{a} \mu(x) d x\right] d a,
$$

where $\mu(x)$ is the force of mortality (hazard of death) at age $x$ as estimated from observed counts of age-specific deaths and the age-specific population at risk. In contrast, life expectancy under current conditions is given by

$$
e_{o}^{*}=\int_{0}^{\omega} \exp \left[-\int_{0}^{a} \mu^{*}(x) d x\right] d a,
$$

where $\mu^{*}(x)$ is the force of mortality that would be experienced by a cohort of newborns that lived all their lives under the mortality regime that prevailed at the time of their birth (Vaupel 2002). Note that this concept of life expectancy under current conditions is a period measure based on the hypothetical notion that the mortality regime at the time of the cohort's birth continues unchanged until the last member of the cohort dies.

It is helpful to consider a special, simple case. Suppose that there is a mortality regime that prevails up until some point in time after which a new mortality regime prevails. Although there may have been human populations in the past that lived under a more or less fixed mortality regime, a sudden but long-term shift from one regime to another may rarely if ever been experienced. In contemporary human populations mortality conditions are changing incessantly. In laboratory experiments, however, with non-human populations, it is possible to switch from one mortality regime to another. For instance, many experiments have been performed with animals such as fruit flies or mice that are given one kind of diet up until some point and a different kind of diet afterwards (e.g., Mair et al. 2003, Carey et al. 1998). The Max Planck Institute for Demographic Research houses a lightbulb laboratory in which large numbers of small 
bulbs can be lit at either 5 or 6 volts. A population of lightbulbs can be kept under harsh 6 volt conditions until some moment and then at a more salubrious 5 volts thereafter.

To simply the exposition, it is also helpful to make three other assumptions. The number of births in the population during each time interval is assumed to be constant. The population is assumed to be closed to migration. And the new mortality regime is assumed to be more favorable than the old regime. In particular, every individual under the new conditions would live at least as long as under the older conditions and some individuals would live longer. Again, such assumptions do not pertain to actual human populations, but they can be achieved in laboratory experiments. So it is possible to imagine a concrete instance of the kind of mortality shift I will consider in this paperthink, e.g., about laboratory flies fed a poor diet and then a better diet or about lightbulbs lit at 6 volts and then at 5 volts. In any case, the assumptions are not of fundamental importance. The theory can be generalized. The assumptions, however, drastically simplify the exposition of the theory.

Consider age-specific death rates in the interval right after the shift from the unfavorable mortality regime to the favorable regime. To be specific, consider lightbulbs during the day after voltage has been lowered from 6 to 5 volts. Suppose that the population of lightbulbs consists of different cohorts that were turned on on different days. Let $\mu(x)$ in formula (1) above be the force of mortality for bulbs that were turned on $\mathrm{x}$ days ago, as estimated from observations of how many bulbs died over the course of the day. Then $e_{o}$, life expectancy at current rates, can be calculated using (1). Suppose a cohort of lightbulbs is illuminated at 5 volts until the last bulb fails. If laboratory conditions are held constant, then observations of this cohort can be used to estimate $\mu^{*}(x)$ in formula (2) and hence $e_{o}^{*}$, life expectancy under current conditions. The main thrust of this article is to demonstrate that, except in special circumstances, $\mu(x)$ will not equal $\mu^{*}(x)$ and hence $e_{o}$ will differ from $e_{o}^{*}$. Annette Baudisch, Jutta Gampe, Mieke Reuser, Dirk Vieregg and I are currently conducting lightbulb experiments to provide empirical evidence for this assertion; in this article I will present the theoretical case.

Vaupel, Manton and Stallard (1979) considered populations of individuals that are heterogeneous with respect to their age-specific chances of death. They showed that if mortality conditions are changing, then life expectancy at current rates does not, except in special circumstances, equal life expectancy under current conditions, i.e., that $e_{o} \neq e_{o}^{*}$. They developed a model of gamma-distributed frailty (i.e., relative risk of death) and used it to derive formulas for $\mu^{*}$ and $e_{o}^{*}$. Further research (e.g., Vaupel and Yashin 1985, 1987a, 1987b; Vaupel, Yashin and Manton 1988; and Vaupel 2002) extends this line of thinking to other kinds of models of heterogeneous populations. The 
fundamental concept of these models is that a cohort's mortality at some age depends not only on current conditions but also on the historical conditions the cohort has suffered.

Bongaarts and Feeney (2002) formulated a delayed-death model such that health improvements in some year add an increment $\delta$ to the remaining lifespans of everyone over 30. Note that this increment does not depend on the mortality history of a population but only on a change in current conditions. In the delayed-death model it is also the case that, except in special circumstances, $e_{o} \neq e_{o}^{*}$. Bongaarts and Feeney $(2002,2003,2005)$ suggest various formulas for estimating $e_{o}^{*}$. Some scholars think that these formulas are problematic (e.g., Wachter 2005). I will not consider this issue here, but I will show that Bongaarts and Feeney's basic point, that $e_{o} \neq e_{o}^{*}$, is correct.

The general conclusion of the present article is that $e_{o} \neq e_{o}^{*}$ (and $\mu(x) \neq \mu^{*}(x)$ ) under much broader circumstances than those considered by Vaupel, Manton and Stallard (1979) or by Bongaarts and Feeney (2002, 2003, 2005). A discrepancy can arise whenever mortality conditions are changing. The two life expectancies will differ when lives saved at age $x$ are extended by an average increment that is not equal to remaining life expectancy at age $x$ at current death rates. Furthermore, the two life expectancies will differ when lives lost at age $x$ are shortened by an average decrement that is not equal to conventionally-calculated remaining life expectancy at age $x$. Bongaarts and Feeney's delayed-death model and the various models of heterogeneity in innate and acquired frailty are special cases.

\section{How saving a life alters life expectancy}

Consider a stylized population with constant age-specific death rates. Suppose that the population is closed to migration and that the number of births each year is constant. Suppose that at some age $x$ in some year $y$ a life is saved. How will the conventional lifetable for that year differ from the lifetable for previous years? The answer is easily derived if the following formula is used:

$$
e_{x}=\frac{D_{x}}{N_{x}} \cdot \frac{1}{2}+\frac{N_{x}-D_{x}}{N_{x}} \cdot\left(1+e_{x+1}\right)
$$

where $e_{x}$ is remaining life expectancy at age $x, N_{x}$ is the number of individuals who celebrate their $x$ th birthday in year $y$ and $D_{x}$ is the number of deaths among those 
individuals. This formula is consistent with conventional lifetable methods and from the values of $e_{x}$ age-specific death rates and other lifetable statistics can be calculated. In this formulation $e_{x}$ is calculated using data for Lexis rhombuses that extend from the start of year $y$ to the end of year $y+1$, but other formulations based on Lexis squares or on Lexis rhombuses that stretch over two years of age and one year of time could also be used.

If a death is averted at age $x$, then

$$
e_{x}^{*}=\frac{D_{x}-1}{N_{x}} \cdot \frac{1}{2}+\frac{N_{x}-\left(D_{x}-1\right)}{N_{x}} \cdot\left(1+e_{x+1}\right) .
$$

Subtracting (3) from (4) yields

$$
e_{x}^{*}-e_{x}=\frac{1}{N_{x}} \cdot\left(0.5+e_{x+1}\right)
$$

If $n_{x}$ deaths are averted, then

$$
e_{x}^{*}-e_{x}=\frac{n_{x}}{N_{x}} \cdot\left(0.5+e_{x+1}\right) .
$$

The expression $0.5+e_{x+1}$ can be interpreted as the remaining life expectancy of someone whose life was saved at age $x$ (under the assumption that such a life is saved, on average, halfway through the year.)

The implication of (5b) is clear and important: conventional lifetable calculations are consistent with the assumption that when lives are saved at some age $x$ the beneficiaries gain, on average, the remaining life expectancy at that age. More precisely, the assumption is that each beneficiary will face the same age-specific hazards of death for the remainder of his or her life as those faced by individuals with lifespans greater than $x$.

The actual average lifespan gained by the resuscitated may, however, be more or less than remaining life expectancy. To be concrete let $0.5+e_{x+1}$ equal ten but suppose that each resuscitated individual dies one year after being saved. Further suppose that $n_{x} / N_{x}$ is one percent. Then conventional calculations yield an increase in remaining life expectancy at age $x$ of a tenth of a year whereas the actual gain is only a hundredth of a year. In other words, conventional calculations lead to a distorted estimate of real life expectancy. 
Before analyzing this distortion further, it is useful to consider mortality improvements at several ages. Suppose that $n_{x}$ deaths are averted at age $x$ and that $n_{x+1}$ deaths are averted at age $x+1$. Then

$$
e_{x+1}^{*}=\frac{D_{x+1}-n_{x+1}}{N_{x+1}} \cdot \frac{1}{2}+\frac{N_{x+1}-\left(D_{x+1}-n_{x+1}\right)}{N_{x+1}} \cdot\left(1+e_{x+2}\right)
$$

and

$$
e_{x}^{*}=\frac{D_{x}-n_{x}}{N_{x}} \cdot \frac{1}{2}+\frac{N_{x}-\left(D_{x}-n_{x}\right)}{N_{x}} \cdot\left(1+e_{x+1}^{*}\right)
$$

Note that $e_{x}^{*}$ depends on $e_{x+1}^{*}$ rather than on $e_{x+1}$. More generally, if mortality improvements are made at older ages, then persons whose lives are saved at some younger age are assumed to gain, on average, the remaining life expectancy at this age taking into account the progress at older ages.

\section{Individual lifetimes}

To further explore why and when $e_{o} \neq e_{o}^{*}$, the notion of individual lifetimes is useful. Let $X_{i}$ be the lifetime (i.e., age at death) of some specific individual $i$. Let $X_{i}(x, y)$ be the total lifetime of this individual if he or she survives to age $x$ in year $y$, under the assumption that the mortality conditions prevailing in year y persist for the rest of the individual's life. Hence $X_{i}\left(0, y_{o}\right)$ is the age at which the individual would die if mortality conditions remain the same as in the individual's year of birth $y_{o}$.

Suppose mortality is being reduced. For simplicity assume that mortality levels remain the same from year $y_{o}$ to just before year $y$ and then fall suddenly. Suppose that individual $i$ survives to age $x$ in year $y$. Let $x^{-}$and $y^{-}$denote the individual's exact age and the exact time just prior to the mortality improvement. Because before year $y$ there is no change in death rates, $X_{i}\left(x^{-}, y^{-}\right)=X_{i}\left(0, y_{o}\right)$. Mortality improvement means that for at least some individuals $X_{i}(x, y)>X_{i}\left(x^{-}, y^{-}\right)$.

Kenneth Wachter suggested a helpful way of thinking about this model. Imagine that each individual is given a ticket at birth that entitles the person to a specific lifespan. This lifespan at the time of birth $y_{o}$ is denoted above by $X_{i}\left(0, y_{o}\right)$. 
Individuals keep their lifespan tickets until time $y$. Some may die before this time. Among the survivors, some and perhaps all individuals get a new ticket with a new lifespan, namely the lifespan denoted above by $X_{i}(x, y)$. Because the new mortality regime is assumed to persist indefinitely, individuals keep their new lifespan until death. Babies born at time $y$ get tickets that are consistent with the new mortality regime. All babies born at any time after $y$ similarly get tickets that are consistent with the new mortality regime. Consider three possibilities.

First, suppose everyone's life is extended by some increment $\delta$ :

$$
X_{i}(x, y)=X_{i}\left(x^{-}, y^{-}\right)+\delta .
$$

This is the elegantly simple model suggested by Bongaarts and Feeney $(2002,2003$, 2005). The increment $\delta$ would be gained not only by everyone alive at time $y$, but also by all future generations. One way to capture this notion is to allow $x$ to be negative. If $x$ is negative, then the person will be born in $x$ years.

Second, suppose that all individuals henceforth age at a slower pace such that time in the future is stretched out by the factor $1+\rho$ :

$$
X_{i}(x, y)=x+(1+\rho)\left(X_{i}\left(x^{-}, y^{-}\right)-x\right) .
$$

This model can also be formulated as:

$$
X_{i}(x, y)=X_{i}\left(x^{-}, y^{-}\right)+\delta_{i},
$$

where

$$
\delta_{i}=\rho \cdot\left(X_{i}\left(x^{-}, y^{-}\right)-x\right) .
$$

Note that the increment $\delta_{i}$ depends on how much longer the individual would have lived under the conditions prevailing before the mortality improvement. As in the case of constant $\delta$, the mortality improvement would benefit future generations as well as those alive at time $y$.

Third, suppose that some individuals gain from the mortality improvement and some do not. In particular, suppose that there is a chance $\pi$ that a specific individual's life will be extended and a corresponding chance $1-\pi$ that the individual's life will not be extended. That is, suppose 


$$
\operatorname{Pr}\left\{X_{i}(x, y)=X_{i}\left(x^{-}, y^{-}\right)+\delta_{i}\right\}=\pi
$$

and

$$
\operatorname{Pr}\left\{X_{i}(x, y)=X_{i}\left(x^{-}, y^{-}\right)\right\}=1-\pi
$$

where $\operatorname{Pr}\{\ldots\}$ denotes "probability of" and the increment $\delta_{i}$ is greater than zero. Further suppose that $\delta_{i}$ is a random variable that has the same distribution as the distribution of remaining lifespans at age $X_{i}$. That is, suppose that when the life is saved of an individual who would have died at some age $X_{i}$, then this individual thereafter faces the same life chances as individuals whose lifespans are greater than $X_{i}$. If $\overline{\delta_{i}}$ is the expected value of $\delta_{i}$, then $\overline{\delta_{i}}$ equals remaining life expectancy at age $X_{i}$. Hence, individuals whose lives are saved at age $X$ gain, on average, the remaining life expectancy at age $X$. This model is consistent with the conventional approach to estimating life expectancy, as shown above.

Note that in all three cases the individual does not have to be on the verge of death. That is, $X_{i}$ may be larger than $x$. A person who would have died at some age in some future year might have the scythe of death averted in an earlier year. Indeed, as noted above, the person might not even be born yet. (If $X_{i}$ is smaller than $x$, then the individual has died and the improvement is too late.)

\section{The triangle of turbulence}

The three special cases described above are illustrative of the range of possibilities. It is also useful to consider a stylized model of the general situation. Consider a hypothetical population that is closed to migration. The number of births each year is constant. Until year 0 an unchanging mortality regime has prevailed for longer than any individual has lived. Then, on January $1^{\text {st }}$ of that year, a new, more favorable mortality regime starts and persists for longer than the maximum lifespan of individuals in the population. Let $D_{y}(x)$ denote the number of deaths over the course of year $\mathrm{y}$ among those who were $\mathrm{x}$ years old on January $1^{\text {st }}$ of that year. Note that $D_{y}(x)$ is the number of individuals such that $x \leq X_{i}(x, y)<x+1$. Let $D_{y}^{o}$ denote the number of deaths of babies who are born in year $\mathrm{y}$ and who die the same year. Assume that for any two years $y_{1}<0, \quad y_{2}<0$, 
when the old mortality regime prevailed, $D_{y_{1}}(x)=D_{y_{2}}(x)$ and $D_{y_{1}}^{o}=D_{y_{2}}^{o}$. (In a study of empirical data, stochastic variation would have to be considered, but the purpose of the stylized model here is cogent exposition rather than statistical analysis.) For cohorts born in any two years, $y_{1} \geq 0, \quad y_{2} \geq 0$, when the new mortality regime prevails, $D_{y_{1}+x}(x)=D_{y_{2}+x}(x)$ and $D_{y_{1}}^{o}=D_{y_{2}}^{o}$. The values of interest lie in a triangle on the $\mathrm{x}, \mathrm{y}$ plane, the triangle bounded by the $\mathrm{x}$ axis at $\mathrm{y}=0$ and by the diagonal cohort line along which $\mathrm{x}=\mathrm{y}$. There can be a turbulent pattern of death counts in this triangle even though the entire triangle lies in the domain of the new mortality regime.

To understand this, consider the number of lives saved in the first year of the new mortality regime among those who are $\mathrm{x}$ years old when the new regime starts on January $1^{\text {st }}$ of year 0 . The number is simply $D_{-1}(x)-D_{0}(x)$. This difference gives the number of individuals who would have died under the old regime who do not die under the new regime. In terms of the ticket concept, it is the number of individuals who get a new ticket that entitles them to a longer life such that they do not die in the year that they would have died if they had not gotten a new ticket. Because the number of individuals who are age $\mathrm{x}$ on January $1^{\text {st }}$ of year 0 equals the number on January $1^{\text {st }}$ of year -1 , the values of $D_{-1}(x)-D_{0}(x)$ determine the gain in conventionally-calculated life expectancy, $e_{o}(0)-e_{o}(-1)$. The number of lives saved, however, does not reveal the increment of additional lifespan that is gained by the resuscitated. As discussed above, conventional calculations assume that the increment is determined by the remaining lifespans of those who lives were not saved but this may not be the case.

In all years after time 0 a constant mortality regime prevails and there is a constant number of births each year and no migration. So why should the number of deaths at some age vary from year to year? In particular, why should the number of deaths at each age in each year not be the same as the number at the age in year 0 ? The reason is that individuals whose lives were saved in year 0 are dying in various years and at various ages and these postponed deaths are adding to the death count. Under the new mortality regime some individuals who would have died after year 0 also are gaining lifespan extensions. As those resuscitated in year 0 and subsequent years die, they add to death counts.

Except in special cases, notably the conventional life expectancy model, the shifting pattern of age-specific death counts will result in changing values of agespecific death rates in the triangle and changing values of conventionally-calculated period life expectancy. The mortality regime changes on January $1^{\text {st }}$ of year 0 , but there is a wake of mortality turbulence that lingers on, gradually diminishing, until it completely peters out after the death of the last individual born under the old mortality regime whose lifespan was extended under the new regime. 


\section{How large is the distortion?}

How distorted is the conventional calculation of life expectancy when death rates are declining? The results above show that the answer depends on how long death is averted. As noted earlier, Bongaarts and Feeney (2002) suggest an ingenious estimate. Let $X_{i}(x-1, y-1)$ be the total lifetime, under the mortality regime prevailing in year $y$ 1 , of an individual who attains age $x-1$ at the start of year $y-1$ and who survives to reach age $x$ at the start of year $y$. Suppose there are improvements in health conditions at the start of year $y$ that benefit everyone equally. That is, suppose the new age at death is

$$
X_{i}(x, y)=X_{i}(x-1, y-1)+\delta, \quad \text { all } i .
$$

Then if deaths are uniformly distributed over the course of a year, the number of lives saved is $n_{x}=\delta \cdot D_{x}$. If, for instance, $\delta$ is 0.25 (i.e., three months), then one-quarter of deaths would be averted (namely, all the deaths from October 1 through December 31. In this view, health improvements delay death equally for everyone.

This is an extreme assumption-and an elegant one. It can be relaxed by letting $\delta$ vary with age and letting $\delta$ vary, in the interval $0 \leq \delta<1$, across individuals.

These are details. The key idea is that annual health progress lets many people gain a short additional span of life. Conventional lifetable calculations, in contrast, are consistent with the notion that lifesaving helps a few people gain (on average) a long increment of life, namely, remaining life expectancy at the age when they would have died.

If deaths are being delayed by a fraction of a year $\delta$ each year, then $e_{o}^{*}(y)$, the true life expectancy at birth in year $y$, is given by

$$
e_{o}^{*}(y)=\int_{0}^{\omega} \exp \left(-\int_{0}^{a} \mu(x, y) /(1-\delta) d x\right) d a .
$$

Note that the starting age 0 might be age 30 or some other age after which the delayeddeath model is assumed to hold. As described above, death counts are assumed to be reduced by the factor $\delta$, so the "observed" force of mortality has to be adjusted by dividing by $1-\delta$. Bongaarts and Feeney suggested several approaches to estimating $\delta$, reviewed by Bongaarts (2005). The validity of the estimates is controversial, as discussed by Wachter (2005). Further research is needed to resolve the issues. It seems likely, however, that if conventionally-calculated life expectancy is increasing, say, at a quarter of a year per year because of a uniform delay in deaths, then $\delta$ is probably 
close to 0.25 . If so, $e_{o}^{*}(y)$ can be two or three years less than $e_{o}(y)$, as argued by Bongaarts and Feeney $(2002,2003)$.

\section{Considerations about true life expectancy}

The true life expectancy of a synthetic cohort living under current mortality conditions might not be as low as the delayed-death model suggests. It might, however, be lower than conventional lifetable calculations imply. The issues here are complicated and require extensive research. Let me adumbrate some key considerations.

If life expectancy in some year is defined as the value implied by current death rates using the conventional formula, then life expectancy cannot be considered distorted. It is simply the value implied by current rates. Demographers, however, usually describe life expectancy as the average lifespan of a synthetic cohort of individuals who live all their lives under current mortality conditions. Life expectancy under current mortality conditions may not be equal to life expectancy calculated using current mortality rates. As explained above, a discrepancy can arise when mortality changes. When death rates are decreasing, then life expectancy under current mortality conditions is less than life expectancy under current mortality rates if those whose deaths are averted have a remaining lifespan that is less than the remaining life expectancy implied by conventional calculations. The bigger the difference between the actual increments in lifespans and lifetable values of remaining life expectancies, the bigger the discrepancy. A similar kind of discrepancy, of opposite sign, can arise when death rates are increasing.

At some ages (e.g., infancy and childhood) most of the lives saved, at least historically and perhaps also today, may have been extended for many years. Bongaarts and Feeney acknowledge this and apply their delayed-death approach only after age 30, after 1950, and in developed countries.

At least some mortality improvements have occurred because specific causes of death have been reduced. To the extent that some people were at higher risk than others, then a fraction of the population gained substantial increments of life. LeBras (2005) discusses this.

Bongaarts and Feeney $(2002,2003)$ show that in developed countries the agespecific force of mortality after age 30 can be approximated by parallel Gompertz curves that shift outward to higher ages over time. This finding is consistent with the delayed-death model and the notion that health progress is helping everyone above 30 more or less uniformly. If lifesaving were due to multiple actions that reduced various causes of death at various ages, then a more complicated pattern of change in agespecific mortality might be expected. 
The notion that general improvements in health conditions are delaying death uniformly for everyone does, however, have an implication that may be questionable in some circumstances. The delayed-death model assumes that everyone benefits from the delay-at all ages (at least above 30) and even if a person would not have died for many years. Hence, a 60 year-old who would otherwise have died this year and a newborn who would have died at age 60 some 60 years from now are both assumed to gain the same extension of life. The newborn, however, will enjoy the improved health conditions for six decades before benefiting whereas the 60 year-old hardly experiences the improved conditions. Whether this is important depends on the nature of the mortality improvement. If, for instance, the improvement is a drug that adds three months between the onset of a disease and death, then the delayed-death model would be appropriate.

Finally, let me highlight an implausible implication of the conventional lifetable approach. The implication was discussed in an article on "Repeated Resuscitation: How Lifesaving Alters Life Tables" (Vaupel and Yashin 1987a). Suppose death rates are lower at every age in some year compared with some previous year. A person who lived his or her entire life under the better mortality conditions might benefit from not dying at the age he or she would have died under the inferior conditions. This person might also benefit at a later age from the lifesaving implied by the lower death rates. Hence, the person might be repeatedly resuscitated. The article provides formulas and several examples. Here let me quote a single example. Consider the simple case such that death rates in the more favorable mortality regime are half as high, at all ages, as in the less favorable regime. Then, as explained by Vaupel and Yashin (1987a), "at the moment death would have occurred, half of the individuals are reprieved - and the other half die as before.... [H]alf of the cohort do not benefit from lifesaving...." That is, life expectancy at birth for those whose lives are not saved is the life expectancy under the unfavorable mortality regime. It seems implausible to me, at least under the conditions that have recently prevailed in developed countries, that mortality improvements that cut death rates in half would be of no direct benefit to half the population. The halving of death rates would be achieved as a result of substantial improvements in health and such improvements would probably, it seems to me, help nearly everyone live at least a bit longer.

These various considerations suggest that both the conventional lifetable approach and the delayed-death model may be extreme cases. On the one hand, it seems unlikely that mortality improvements result in death being averted for a few people who gain, on average, remaining life expectancy. Those whose lives are saved probably tend to be relatively frail or vulnerable and their remaining lifetimes are probably, on average, shorter than the remaining life expectancy of those not rescued from death. On the other hand, it also seems unlikely that mortality improvements give everyone the same 
lifespan increment, regardless of age and regardless of how long an individual will live under the new health regime. It is therefore useful to consider other models. One approach is provided by models of heterogeneity in innate or acquired frailty, as I discussed in an earlier reflexion on Bongaarts and Feeney's contributions (Vaupel, 2002). Another approach is sketched below.

\section{A model of stretched lifetimes}

For cold-blooded animals life runs more slowly when temperatures are lower. In particular, the trajectory of age-specific death rates is stretched out over a longer period of time at lower vs. higher temperatures (Mair, Goymer, Pletcher, Partridge 2003). Reliability engineers use "accelerated-failure-time models" to describe this kind of phenomenon. It is a misleading term from the perspective of this article because the focus here is on reductions rather than increases in mortality. Hence, I will refer to "stretched-lifetime models". The basic idea is that the deaths that would have occurred over some period of time $t$ occur over a longer period of time $(1+\rho) t$. This model is summarized above in formulas (4a), (4b) and (4c).

Consider the simple case when $\rho$ is one. If life expectancy was $e_{o}$ before the mortality improvement, then it is $2 \cdot e_{o}$ after the improvement. That is, a cohort living under the new conditions will live twice as long on average. From a period perspective, the number of deaths in some time interval following the improvement will be half as many as the number of deaths in a similar time interval before the improvement. Hence, conventional lifetable calculations will be based on death rates that have been cut by a factor of two. If death rates are the same at all ages, then cutting death rates by a factor of two is equivalent to stretching time by a factor of two. But if death rates change over age, this is not the case. In some countries women suffer about half the age-specific chance of death as men, but in these countries women do not live twice as long as men but only about six years longer. In the case of stretched lifespans when mortality increases with age, conventional lifetable calculations underestimate true life expectancy.

\section{Quantum and tempo vs. proportions and increments}

So far, the word tempo has not appeared in this article. This may seem strange in a contribution, inspired by the work by Bongaarts and Feeney on tempo distortions, that is appearing in a collection of articles on tempo effects on mortality. The notion that 
there are quantum and tempo effects on fertility was developed by Norman Ryder: quantum refers to the number of births and tempo to the pace of childbearing. For demographers deeply versed in this tradition it may be useful to consider lifesaving from a tempo perspective. The concept of tempo effects on fertility stimulated Bongaarts and Feeney to question conventional calculations of life expectancy. It seems to me, however, that the tempo-quantum metaphor may sometimes be more misleading than helpful, at least in studies of mortality, and that the metaphor has to be treated with great caution.

To my mind, a better starting point is the fact that all populations are heterogeneous and that changes in conditions will affect individuals differently. If mortality is reduced, then some individuals may gain no additional life, some may gain a little, and some may gain a lot. So a natural vantage point for me is to think (e.g., as in Vaupel 2002) about what proportion of people gain how much. In the case of reductions in period fertility, it may similarly be useful to consider how many women (and men) are postponing childbearing by how long (and how many are choosing not to have an additional child at all.)

The three cases considered in this article - the conventional lifetable approach, the delayed-death model, and the stretched-lifetime model—neglect heterogeneity. In the conventional approach, all individuals resuscitated at some age, together with all individuals who would otherwise have survived this age, face the same schedule of mortality at subsequent ages. The delayed-death model assumes that all individuals gain the same increment of life. The stretched-lifetime model slows time equally for everyone. Because all populations are heterogeneous, all three perspectives are wrong. Nonetheless, all three perspectives may prove useful. Each of the models may provide serviceable approximations or bounds for at least some kinds of lifesaving interventions in some contexts. Alternative models that build on the theory of heterogeneous populations may also prove useful.

\section{Directions for research}

The conventional lifetable approach, the delayed-death model, the stretched-lifetime model, and various models of innate and acquired heterogeneity may all offer useful perspectives for understanding the fundamental nature of mortality change. Some kinds of change may extend a few people's lives for an average period that may approach remaining life expectancy. Other kinds of change may extend many people's lives for a short time. Still other kinds of change may slow the clock of aging. All populations are heterogeneous, so each of these kinds of changes may affect individuals somewhat differently. Hence it is a question of both/and rather than either/or. That is, research is 
not needed to determine which model is right and which models are wrong. All the models are wrong. The research required is research to determine which model or mix of models is most helpful in understanding mortality change at various ages, various times, and as a result of various kinds of interventions.

There are two directions for this research: theoretical thinking about the nature of lifesaving and empirical analyses that test alternative theoretical hypotheses. This article and previously published studies about tempo effects on mortality and on the theory of heterogeneous populations have contributed to theoretical understanding. Great progress has been made in understanding how lifesaving may, theoretically, affect lifetimes and lifetables. The burst of innovation since Bongaarts' and Feeney's (2002) stimulating insight is greater than that in any comparable period since 1662, when Graunt's seminal study was published. Theoretical research on the impact of lifesaving is burgeoning with vitality and fresh new growth. Further thinking will almost certainly produce a spate of further ideas.

As noted in the introduction, the basic thrust of this article is that life expectancy at current rates will generally differ from life expectancy under current conditions when the following two conditions both hold. First, current mortality conditions differ from past mortality conditions. Second, the lives saved or lost at each age $x$ (because of the change in mortality conditions) are extended or shortened by an average amount that is not equal to remaining life expectancy at age $x$ at current death rates. There may be special circumstances when the second condition is true but the two life expectancies turn out to be the same. In particular, at some ages $x$ lives might be saved for less than remaining life expectancy and at other ages by more and the effects might cancel out. Such a coincidence, however, will be unusual. Bongaarts' and Feeney's delayed-death model is a special, extreme case of the second condition. Other cases include the stretched-lifetime model and various heterogeneity models. The heterogeneity among individuals does not have to be fixed and innate: the heterogeneity can be acquired as individuals experience various events that weaken or strengthen them.

Because the phrase "tempo distortions of mortality" is generally used with reference to the delayed-death model, some broader phrase should be used to describe the general fact that when mortality is changing conventional lifetables do not, except in special circumstances, describe the age-trajectory of mortality that a cohort would experience under current conditions. Let me suggest use of the phrase "the theory of mortality turbulence" to allude to the general phenomenon. The notion is that, except in special cases, mortality change creates a wake of turbulence, of disequilibrium, that temporarily distorts death rates. In this case, the current rates may not equal the death rates that will eventually prevail when the turmoil ceases.

The turbulence could be due to various factors. In addition to delays in death, stretched lifespans, and the impact of differential mortality in heterogeneous 
populations, it may be the case that the longer individuals have lived under the unfavorable mortality regime, the more debilitated they are and the less they are to benefit from more favorable conditions. On the other hand, individuals who have survived the old regime may have been strengthened (in terms, say, of immune response). Such "hormesis" may enable them to benefit more from the new regime than cohorts born under the new regime. The shift in mortality regime may be disturbing to individuals: they may need time to adapt to the new conditions. For example, animals brought from the wild into a zoo or laboratory may suffer especially high mortality during an initial period.

A broader theory of demographic disturbance might be developed to study transient distortions produced by fertility change, marriage change, etc. Bongaarts and Feeney (2005) argue that tempo distortions influence a variety of demographic rates. Similarly and more generally, there may be a wake of turbulence following change in fertility, marriage and other demographic regimes. Such turbulence will occur if period statistics are based on data that reflect how many events (e.g., births or marriages) are averted in the period but do not capture the length of time the events are postponed.

Empirical research is required to test hypotheses arising from the theory of mortality turbulence. Data on cohort mortality can be used to distinguish between interventions that stretch lifetimes vs. those that lower age-specific mortality but do not decelerate the rate of increase in death rates with age (Mair et al. 2002). What are urgently needed are empirical tests that distinguish between the conventional view of lifesaving and the Bongaarts-Feeney delayed-death model. It seems clear that both perspectives are wrong and that true period life expectancy probably lies somewhere between the conventional estimate and a delayed-death estimate. But where inbetween? It may well be that for remaining life expectancy at age 30 in developed countries since 1950 Bongaarts and Feeney are closer to the truth. Indirectly relevant evidence can be advanced to support their position. The discussion, however, will remain speculative until direct tests can be developed. This is the key challenge today for basic research on mortality.

The lightbulb experiments being conducted at the Max Planck Institute for Demographic Research are a first step. Experiments with various animal models, flies or nematode worms for instance, will also be important. As theoretical understanding of mortality turbulence develops and as empirical results are found in laboratory experiments, then it may become possible to develop and refine strategies for analyzing human data. If the Bongaarts-Feeney delayed death model is consistent with the results of laboratory experiments, then it might also hold for human populations under some circumstances. More generally, the lightbulb and other experiments will help illuminate how the terrain of the triangle of mortality turbulence is shaped by different kinds of mortality change. 


\section{Acknowledgements}

For constructive discussions and comments, I thank Kenneth Wachter, John Bongaarts

and Griff Feeney, Annette Baudisch, Jutta Gampe and Elisabetta Barbi, and two anonymous reviewers. 


\section{References}

Bongaarts, J. (2005) "Five Period Measures of Longevity" Demographic Research, Volume 13, Article 21; http://www.demographic-research.org

Bongaarts, J. and G. Feeney (2002) "How Long Do We Live?", Population and Development Review 28(1): 13-29.

Bongaarts, J. and G. Feeney (2003) "Estimating mean lifetime," Proceedings of the National Academy of Sciences 100(23): 13127-13133.

Bongaarts, J. and Feeney, G.. (2005). The quantum and tempo of life cycle events. Policy Research Division Working Paper No. 207. New York City: Population Council. http://www.popcouncil.org/.

Carey J.R., P. Liedo, H.-G. Müller, J.-L. Wang and J.W. Vaupel (1998) "Dual modes of aging in Mediterranean Fruit Fly females", Science. 281: 5379: 996-998.

LeBras, H. (2005) "How Behavioural Changes Are Reflected in the Changes of Mortality Measurements" Forthcoming, Demographic Research, http://www.demographic-research.org

Mair, W., P. Goymer, S.D. Pletcher, L. Partridge (2003) "Demography of Dietary Restriction and Death in Drosophila", Science 301: 1731-1733.

Vaupel, J.W. (2002) "Life Expectancy at Current Rates vs. Current Conditions", Demographic Research 7(8): 366-376.

Vaupel, J.W., K.G. Manton and E. Stallard (1979) "The Impact of Heterogeneity in Individual Frailty on the Dynamics of Mortality", Demography 16: 439-454.

Vaupel J.W. and A.I. Yashin (1985) "Heterogeneity's Ruses: Some Surprising Effects of Selection on Population Dynamics", American Statistician 39(3): 176-85.

Vaupel J.W. and A.I. Yashin (1987a) "Repeated Resuscitation: How Lifesaving Alters Life Tables", Demography. 24(1): 123-135.

Vaupel J.W. and A.I. Yashin (1987b) "Targeting Lifesaving: Demographic Linkages between Population structure and Life Expectancy", European Journal of Population 2(3-4): 335-360.

Vaupel J.W., A.I. Yashin and K.G. Manton (1988) "Debilitation's Aftermath: Stochastic Process Models of Mortality", Mathematical Populations Studies 1(1): 21-48.

Wachter, K.W. (2005) “Tempo and its Tribulations" Demographic Research, Volume 13, Article 9; http://www.demographic-research.org 